\title{
Perceived Barriers in the Use of Point of Care Ultrasound in the WWAMI Region
}

Keywords: Emergency medicine; Rural; Ultrasound; Point-of-care

\section{Abstract}

Objective: The use of point-of-care ultrasound (POCUS) has increased in use over the last two decades. Despite reports of increased use of POCUS at academic emergency departments, the overall trends in use of POCUS in rural emergency departments remains low. We aimed to define the perceived barriers to the use of emergency POCUS by physicians in the five-state WWAMI region (Washington, Wyoming, Alaska, Montana, and Idaho).

Method: A 26-question survey was sent to emergency physician who practice in the WWAMI region. This was a convenience sample obtained from list-serves of emergency medicine and emergency ultrasound societies. The survey was analyzed and descriptive results were obtains.

Results: We sent our survey to a total of 353 providers. 65 respondents completed the survey, for a response rate of $18.4 \%$. Most physicians reported their clinical practice site as being in Alaska Washington or Idaho, and nearly all of them (96\%) had access to an ultrasound machine owned by the emergency department group. $57 \%$ of respondents work in an emergency department with an annual census of less than 40,000 patients per year. $60 \%$ noted having participated in emergency POCUS after their residency training period, most commonly through a continuing medical education course. Participants were most confident performing emergency POCUS for trauma (FAST exam) and using POCUS for procedural guidance. Respondents noted being less confident performing POCUS for focused echocardiography, biliary, lung and intravascular volume assessment. The most commonly identified barriers to using emergency POCUS were concerns about liability, difficulty with image interpretation, lack of training, and lack of POCUS support personnel.

Conclusions: Emergency physicians from the WWAMI state identify lack of training as a primary barrier to regular use of pointof-care ultrasound in their practice. Most participants identified telesonography and web-based distance learning as methods to enhance their POCUS education.

\section{Introduction}

Point-of-care ultrasound (POCUS) is defined as a focused, goal-oriented bedside ultrasound examination performed by the treating physician to answer a specific question or guide an invasive procedure. POCUS is most frequently performed by physicians from emergency medicine, intensive care, surgical specialties and family medicine, among others [1]. Current literature has demonstrated high accuracy rates, improved outcomes and shortened time to operative intervention when an ultrasound examination is included in the initial evaluation of the unstable trauma patient [2-6]. Ultrasoundguided central venous cannulation improves success rate, decreases access time, and reduces complications [7-10]. Patients report higher satisfaction with their emergency physician, diagnostic testing, and overall ED care when POCUS is used [11]. The healthcare settings in which POCUS is performed, and the specialties that routinely use POCUS, have grown dramatically over the past ten years [12-14].

\section{Journal of}

Emergency Medicine \& Critical Care

\author{
Blaise Bellows, Jodie Totten, Sachita Shah, and \\ Adeyinka Adedipe* \\ Division of Emergency Medicine, Department of Medicine, \\ University of Washington, WA, USA
}

\section{Address for Correspondence}

Adeyinka Adedipe, MD RDMS, Division of Emergency Medicine, Department of Medicine, University of Washington, 325 9th Avenue, Box \#359702 Seattle, WA 98104, USA, Tel: 206.744.8459; E-mail: adeyinka@uw.edu

\section{Submission: 05 March, 2015}

Accepted: 16 March, 2015

Published: 21 March, 2015

Reviewed \& Approved by: Dr. Christopher R. Tainter, Assistant Professor, Department of Emergency Medicine, University of California, USA

The use of POCUS in non-academic hospital systems has seen a marked increase largely due to ease of learning, high accuracy rates, availability, and enhanced portability associated with POCUS $[15,16]$. Point-of-care ultrasound devices have become smaller, less expensive, and more durable, making them a very attractive option for areas with limited resources. Rural areas in the United States have the potential to implement novel technology such as POCUS, and leverage their relative proximity to regional training institutions for guidance. Although ultrasound is readily available, there may be challenges to using point-of-care ultrasound for providers in less populous states. Much of the current literature on emergency point-ofcare ultrasound addresses implementation and training of ultrasound in the developing world. Few studies have specifically addressed the challenges faced by rural providers in developed nations [17-20]. We sought to define the perceived barriers of point-of-care ultrasound in the rural areas served by our training institution. More than 50 million people in the United States live in rural areas, defined by the US Census Bureau as tracts outside of urban clusters with $>2,500$ population and lying outside of "urbanized areas" [21].

The University of Washington in Seattle is the regional referral center for the five state regions known as "WWAMI." The WWAMI region encompasses the states of Washington, Wyoming, Alaska, Montana and Idaho, collectively a mostly rural area representing nearly $25 \%$ of the United States' land mass. Nearly one quarter of WWAMI states' combined populations live in rural areas [22].

Our goal was to assess perceived barriers to the use of point-ofcareultrasoundbyemergencyphysiciansintheWWAMIstates.Inaddition, we sought to investigate provider interest in distance learning in emergency point-of-care ultrasound.

\section{Methods}

We conducted a survey of emergency physicians from the WWAMI states, a convenience sample obtained from list-serves of emergency medicine and emergency ultrasound societies. We developed a 26-question online survey that contained demograph- 
ic questions including prior POCUS training, type of residency training, clinical practice, and zip code of the study participant (Appendix). The types of POCUS exams performed and available configuration of transducers was ascertained. Study participants were asked to rank their level of confidence in performing and interpreting the primary emergency POCUS applications (e.g. FAST, cardiac, early pregnancy). Several questions related to the barriers to performing POCUS, such as hardware and technical issues, machine availability and decision support. Additional questions related to the capabilities of the clinical practice environment, should a POCUS result warrant further subspecialty support from radiology or surgery.

The survey was analyzed, and descriptive results were obtained from multiple-choice and free text answer choices.

\section{Results}

The survey was sent to a total of 353 providers. 65 respondents completed the survey, for a response rate of $18.4 \%$ (Table 1). Most respondents identified their clinical practice as being in the states of Alaska, Washington or Idaho. 49 (75\%) worked in a hospital that was identified as a University of Washington affiliate site, and 61 (94\%) considered their site a community practice. 37 (57\%) respondents described their site's annual census as less than 40,000 patients per year. $55(85 \%)$ completed a residency in emergency medicine, and $38(59 \%)$ were more than 10 years post-graduation from residency training. $39(60 \%)$ respondents completed training in emergency POCUS outside of training, predominantly via continuing medical education course.

Nearly all respondents (96\%) have an ultrasound machine in the emergency department that is owned by their emergency department group. Of those who responded in the affirmative, $100 \%$ had linear transducers, and $84 \%$ had curved transducers, with $62 \%$ having an endocavitary transducer and $49 \%$ owning a phased array transducer for cardiac imaging.

Study participants were most confident performing emergency POCUS for trauma (68\% as confident to completely confident) and using POCUS for procedural guidance ( $71 \%$ as confident to completely confident). Study participants were least confident

Table 1: Demographics of Respondents.

\begin{tabular}{|c|c|}
\hline Residency, \% & \\
EM & 85 \\
Other & 15 \\
\hline Years since Graduation, \% & 12 \\
$<5 y r$ & 29 \\
$5-10 y r$ & 59 \\
$>10 y r$ & \\
\hline Practice Type, \% & 94 \\
Community & 6 \\
\hline Academic & \\
\hline UW Affiliate, \% & 75 \\
Yes & 25 \\
No & \\
\hline Annual Volume, \% & 25 \\
$20-40$ & 32 \\
$40-60$ & 25 \\
$60-80$ & 14 \\
$>80$ & 4 \\
\hline
\end{tabular}

performing emergency POCUS for focused cardiac (20\% were not at all or not very confident), lung (20\% were not at all or not very confident) and IVC for volume assessment $(29.8 \%$ not at all or not very confident) (Figure 1). POCUS exams least likely to be performed by study participants were ultrasound assessment for DVT, renal and lung disease.

Several barriers to using POCUS by emergency physicians in the WWAMI region were identified. The most commonly identified barriers related to concerns about liability (33\%), lack of training (62\%), difficulty with image interpretation (35\%), and lack of support personnel (41\%). The least common barriers included lack of internet (11\%), machine malfunction (12\%), and lack of reliable machine maintenance (14\%) (Figure 2). Most respondents noted that their group was not billing for their emergency POCUS studies (70\%), and most respondents stated that they did not have a formal quality assurance process for image review (63\%).

Most study respondents were interested in learning emergency POCUS if a course was taught near to where they lived (80\%), and many expressed interest in participating in a distance learning program for emergency POCUS (64\%). 76\% of respondents felt the instructor should be physically present during the hands-on demonstration. While the majority of respondents strongly agreed that hands-on training sessions were essential to learning bedside ultrasound (84\%), a significant number felt they could learn the principles of bedside ultrasound and hands-on instruction virtually (62\% and $42 \%$ respectively).

\section{Discussion}

The use of point-of-care ultrasound in resource-limited settings outside of the US, and rural areas in the US, has increased over the past two decades. Part of this rapid adoption of POCUS is technology-related (small, portable and durable ultrasound machines are commonplace), while another key factor is practice-related (improved patient care, ease of learning, among others). Despite the availability of bedside ultrasound machines, rural emergency physicians continue to identify significant barriers to integrating POCUS into patient care.

Traditional barriers to implementation of a comprehensive emergency ultrasound program have been previously identified [23-25]. Training guidelines recommend a minimum number of examinations to demonstrate competency, which may be challenging for practicing physicians to complete. A physician knowledgeable in emergency POCUS should be available to provide decision support, direct training, and timely quality assurance. Lastly, the cost to start up and maintain a robust emergency POCUS program may be high for some groups. WWAMI emergency physicians primarily identified lack of training, lack of decision support, and difficulty with image interpretation as the main barriers to using emergency POCUS in their daily practice. Theoretically, many of these barriers, if not all, could be overcome with currently available technology such as real-time video conferencing, email and web-based training modules. While concerns about liability with use of POCUS in the emergency department persist, a review by Blaivas et al. found only one lawsuit filed against an emergency physician over a twenty-year period related to bedside ultrasound, and it was for failure to perform an ultrasound [26]. 
Citation: Bellows B, Totten J, Shah S, Adedipe A. Perceived Barriers in the Use of Point of Care Ultrasound in the WWAMI Region. J Emerg Med Critical Care. 2015;1(1): 4.

ISSN: $2469-4045$

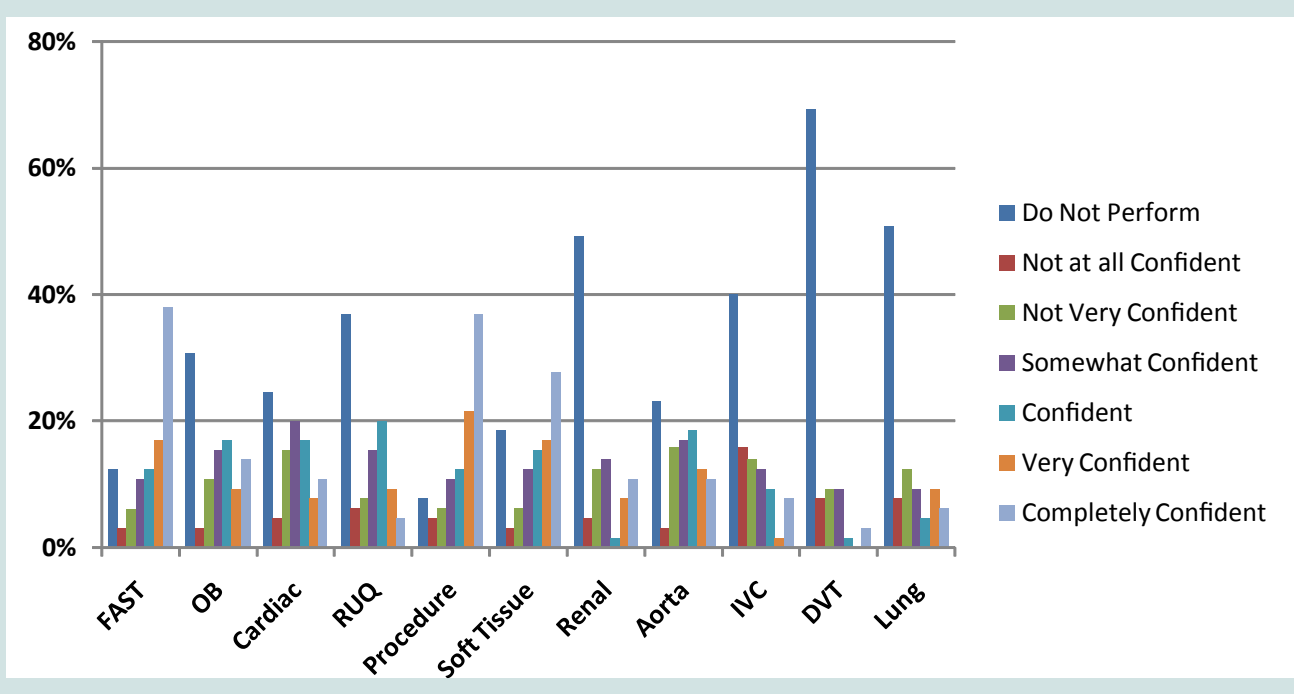

Figure 1: Respondent Confidence by POCUS Exam Type.

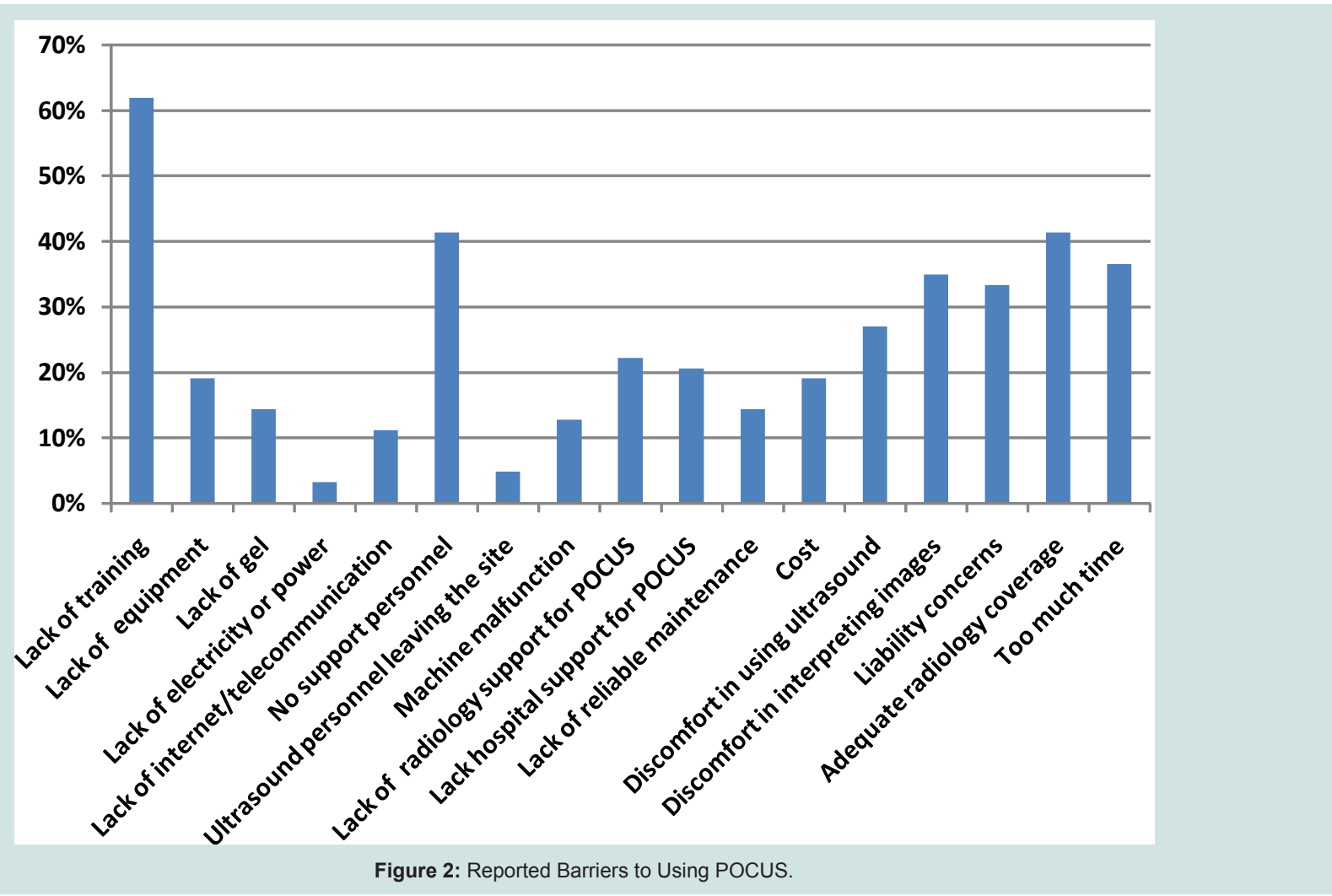

There were several limitations in our study. First, we had a low response rate and small sample size with which to conduct analysis. Specifically, we noted a low response rate from emergency physicians in Montana and Wyoming. Second, our survey may not have reflected the true variety of physicians who provide emergency care, such as those who are not board certified in emergency medicine. The results of this study may also be limited by the use of a convenience sample, a group that may self-select for enthusiasm in the use of ultrasound. Lastly, the survey may be limited by recall bias due to self-reporting.

\section{Conclusion}

Emergency physicians in the WWAMI region identify several barriers to using emergency POCUS routinely in their practice. POCUS is dependent on the skills of the operator and availability of physician support for image interpretation. Our study identified lack of ultrasound training and lack of qualified personnel to answer questions as current barriers to ultrasound use in the WWAMI region. We anticipate using our results to develop an emergency point-of-care ultrasound curriculum designed specifically for the 
Citation: Bellows B, Totten J, Shah S, Adedipe A. Perceived Barriers in the Use of Point of Care Ultrasound in the WWAMI Region. J Emerg Med Critical Care. 2015;1(1): 4.

ISSN: 2469-4045

needs of emergency physicians in the five-state WWAMI region.

\section{References}

1. Moore CL, Copel JA (2011) Point-of-care ultrasonography. N Engl J Med 364 749-757.

2. Ma OJ, Mateer M, Ogata M, Kefer MP, Wittmann D, et al. (1995) Prospective analysis of rapid trauma ultrasound examination performed by emergency physicians. J Trauma 38: 879-895.

3. Rozycki GS, Ballard RB, Feliciano DV, Schmidt JA, Pennington SD (1998) Surgeon-performed ultrasound for the assessment of truncal injuries. Lessons learned from 1540 patients. Ann Surg 228: 557-567.

4. Wherrett LJ, Boulanger BR, McLellan BA, Brenneman FD, Rizoli SB, et al. (1996) Hypotension after blunt abdominal trauma: the role of emergent abdominal sonography in surgical triage. J Trauma 41: 815-820.

5. Boulanger BR, McLellan BA, Brenneman FD, Ochoa J, Kirkpatrick AW (1999) Prospective evidence of the superiority of a sonography-based algorithm in the assessment of blunt abdominal injury. J Trauma 47: 632-637.

6. Diercks DB, Mehrotra A, Nazarian DJ, Promes SB, Decker WW, et al. (2011) Clinical Policy: critical issues in the evaluation of adult patients presenting to the emergency department with acute blunt abdominal trauma. Ann Emerg Med 57: 387-404.

7. Denys BG, Uretsky BF, Reddy PS (1993) Ultrasound-assisted cannulation of the internal jugular vein. A prospective comparison to the external landmarkguided technique. Circulation 87: 1557-1562.

8. Karakitsos D, Labropoulos N, De Groot E, Patrianakos AP, Kouraklis G, et al. (2006) Real-time ultrasound-guided catheterization on the internal jugula vein: a prospective comparison with the landmark technique in critical care patients. Crit Care 10: R162.

9. Leung J, Duffy M, Finckh A (2006) Real-time ultrasonographically-guided internal jugular vein catheterization in the emergency department increases success rates and reduces complications: a randomized, prospective study. Ann Emerg Med 48: 540-547.

10. Kumar A, Chuan A (2009) Ultrasound guided vascular access: efficacy and safety. Best Pract Res Clin Anaesthesiol 23: 299-311.

11. Howard ZD, Noble VE, Marill KA, Sajed D, Rodrigues M, et al. (2014) Bedside ultrasound maximizes patient satisfaction. J Emerg Med 46: 46-53.

12. Fagenholz PJ, Gutman JA, Murray AF, Noble VE, Thomas SH, et al. (2007) Chest ultrasonography for the diagnosis and monitoring of high altitude pulmonary edema. Chest 131: 1013-1018.

13. McNeil CR, McManus J, Mehta S (2009) The accuracy of portable ultrasonography to diagnose fractures in an austere environment. Prehosp Emerg Care 13: 50-52.
14. Shah SP, Epino H, Bukhman G, Umulisa I, Dushimiyimana JM, et al. (2009) Impact of the introduction of ultrasound services in a limited resource setting: rural Rwanda 2008. BMC Int Health Hum Rights 27: 9

15. Stein JC, River G, Kalika I, Hebig A, Price D, et al. (2009) A survey of bedside ultrasound use by emergency physicians in California. J Ultrasound Med 28: 757-763

16. Moore CL, Molina AA, Lin H (2006) Ultrasonography in community emergency departments in the United States: access to ultrasonography performed by consultants and status of emergency physician-performed ultrasonography. Ann Emerg Med 47: 147-153.

17. Lyon M, Blaivas M, Brannam L (2005) Use of emergency ultrasound in a rural ED with limited radiology services. Am J Emerg Med 23: 212-214.

18. Glazebrook R, Manahan D, Chater AB (2005) Evaluation of an ultrasound program (intermediate obstetric and emergency medicine) for Australian rural and remote doctors. Aust J Rural Health 13: 295-299.

19. Flynn CJ, Weppler A, Theodoro D, Haney E, Milne WK (2012) Emergency medicine ultrasonography in rural communities. Can J Rural Med 17: 99-104.

20. Minardi J, Davidov D, Denne N, Haggerty T, Kiefer C, et al. (2013) Bedside ultrasound: advanced technology to improve rural healthcare. $\mathrm{W} \vee \mathrm{V}$ Med $\mathrm{J}$ 109: 28-33.

21. http://www.census.gov/geo/www/ua/ua

22. Skillman SM, Patterson DG, Lishner DM, Doescher MP (2013) The rural health workforce: data and issues for policymakers in Washington, Wyoming, Alaska, Montana, Idaho. Issue \#4: what is rural in the WWAMI States? Why definitions matter. Policy Brief \#146. 4. Seattle, WA: WWAMI Rural Health Research Center, University of Washington.

23. (2008) Policy statement "Emergency Ultrasound Guidelines". American College of Emergency Physicians.

24. Liebeskind ME, Arger PH, Liebeskind A, Maston K, Langlotz C (2002) Using sonography to examine adult patients at an academic medical center: have usage patterns changed with the expansion of managed care? AJR Am J Roentgenol 179: 1395-1399.

25. Shah S1, Noble VE, Umulisa I, Dushimiyimana JM, Bukhman G, et al. (2008) Development of an ultrasound training curriculum in a limited resource international setting: successes and challenges of ultrasound training in rural Rwanda. Int J Emerg Med 1: 193-196.

26. Blaivas M, Pawl R (2012) Analysis of lawsuits filed against emergency physicians for point-of-care emergency ultrasound examination performance and interpretation over a 20-year period. Am J Emerg Med 30: 338-341. 